\title{
Optimal Number of Systematic Biopsy Cores Used in Magnetic Resonance Imaging/Transrectal Ultrasound Fusion Targeted Prostate Biopsy
}

\author{
Shogo Teraoka," Masashi Honda," Ryutaro Shimizu," Ryoma Nishikawa,* Yusuke Kimura," Tetsuya Yumioka,"* \\ Hideto Iwamoto,* Shuichi Morizane,* Katsuya Hikita* and Atsushi Takenaka* \\ *Division of Urology, Department of Surgery, School of Medicine, Faculty of Medicine, Tottori University, Yonago 683-8503, Japan
}

\section{ABSTRACT}

Background In recent years, the effectiveness of magnetic resonance imaging (MRI)-ultrasound fusion targeted biopsy (MRF-TB) has been widely reported. In this study, we assessed the effect of reduction of the number of systematic biopsy (SB) cores on the cancer detection rate (CDR).

Methods Patients with a high prostate-specific antigen (PSA) level underwent prostate MRI. The Prostate Imaging-Reporting and Data System version 2 (PI-RADS) was then used to rate the lesions. The inclusion criteria were as follows: (1) PSA level between 4.0 and $30.0 \mathrm{ng} / \mathrm{mL}$ and (2) patients with one or more lesions on MRI and a PI-RADS score of 3 or more. All enrolled patients were SB naïve or had a history of one or more prior negative SBs. A total of 104 Japanese met this selection criterion. We have traditionally performed 14-core SB following the MRF-TB. In this study, the CDRs of 10core SB methods, excluding biopsy results at the center of the base and mid-level on both sides, were compared with those of the conventional biopsy method.

Results We compared CDRs of the 14-core and 10core SBs used in combination. The overall CDR was $55.8 \%$ for the former and $55.8 \%$ for the latter, thereby indicating that there was no significant difference $(P=$ 1.00) between the two. In addition, the CDRs of csPCa were $51.9 \%$ for the former and $51.1 \%$ for the latter, which indicated that there was no significant difference $(P=$ $0.317)$.

Conclusion There was no significant difference in the $\mathrm{CDR}$ when the number of SB cores to be used in combination was 14 and 10.

\footnotetext{
Corresponding author: Shogo Teraoka, MD teraoka-1119@tottori-u.ac.jp Received 2021 March 16

Accepted 2021 June 4

Online published 2021 July 9

Abbreviations: CDR, cancer detection rate; cisPCa, clinically insignificant $\mathrm{PCa}$; csPCa, clinically significant IQR, interquartile range; PCa; MRI, magnetic resonance imaging; MRF-TB, MRITRUS fusion targeted biopsy; PIN, Prostatic intraepithelial neoplasia; PI-RADS, Prostate Imaging-Reporting and Data System version 2; PSA, prostate specific antigen; $\mathrm{PCa}$, prostate cancer; $\mathrm{SB}$, systematic biopsy; TB, targeted biopsy; TRUS, transrectal ultrasound
}

Key words Image-guided biopsy; magnetic resonance imaging; prostate; prostatic neoplasms; ultrasonography

Prostate biopsy is essential for diagnosis, risk stratification, and treatment planning in patients with prostate cancer (PCa). Only $13 \%$ to $33 \%$ of PCa cases manifest as a single lesion in the prostate. In most cases, $\mathrm{PCa}$ lesions occur in multiple forms. ${ }^{1}$ Therefore, systemic biopsy (SB) has emerged as a useful diagnostic and prognostic tool. However, SB has several limitations. For instance, SB-guided transrectal ultrasound (TRUS) may lead to the underdiagnosis of clinically significant prostate cancer (csPCa) or may result in the overdiagnosis of clinically insignificant $\mathrm{PCa}$ (cisPCa). The overdiagnosis and overtreatment of $\mathrm{PCa}$ have become a serious clinical problem. Hence, new methods are required to improve the accuracy of prostate biopsy.

In the recent years, the usefulness of multiparametric magnetic resonance imaging (mpMRI) in diagnosing csPCa has been reported. In particular, the location of cancer noted on an mpMRI image is fused with the TRUS image using a software. A fusion image is formed, which then guides targeted biopsy (TB). This method is known as the MRI-TRUS fusion-targeted biopsy (MRF-TB). Several meta-analyses have reported that MRF-TB had a higher detection rate of csPCa and a lower number of required biopsy samples compared with TRUS biopsy. ${ }^{2-5}$

Many medical institutions use TB and SB in combination, but the exact number of biopsy cores required in each procedure remains unknown. The number of biopsy cores is determined at the discretion of the examiner based on the size and position of the lesion and the Prostate Imaging-Reporting and Data System version 2 (PI-RADS) score. Our facility performed a 2-core TB and a 14-core SB for each MRI lesion. However, there were numerous reports that a higher number of cores in prostate biopsy not only took more time, but also increased the incidence of complications. Reducing the number of biopsy cores may reduce patient burden.

In this study, the PI-RADS score was used for MRI evaluation of PCa. PI-RADS corresponded to the patient's likelihood of having csPCa. It was based on a combination of mpMRI findings on a 5-point scale. 
Other findings such as prostate-specific antigen (PSA) levels, rectal findings, medical history, or treatment strategy were not accounted in the PI-RADS score.

We assessed the performance of PI-RADS in PCa detection. The PI-RADS score was retrospectively assigned. Furthermore, we evaluated whether the cancer detection rate (CDR) would be the same even if the number of biopsy cores used in the conventional 14core SBs at our institution was reduced to 10 -core. We compared the 14-core and 10-core SBs in terms of their $\mathrm{PCa}$ detection rates.

\section{MATERIALS AND METHODS}

Ethical approval for the collection and analysis of the data was obtained from the Ethics Committee of Tottori University Faculty of Medicine, Yonago, Japan (approval number 20A016). Since this study uses only medical data and other information, the details of the study were disclosed on the website in advance in accordance with the ethical guidelines set by the government. Informed consent is waived by ethics committee. (Tottori University Faculty of Medicine Ethics Review Committee)

\section{Patient selection}

Patients with a high PSA level underwent a 1.5 - or 3-Tesla prostate MRI. The PI-RADS was then used to rate the lesions. The inclusion criteria were as follows: (1) PSA level between 4.0 and $30.0 \mathrm{ng} / \mathrm{mL}$ and (2) patients with one or more lesions on MRI and a PI-RADS score of 3 or more. All enrolled patients were SB naïve or had a history of one or more prior negative SBs. None of the patients had a history of prior MRF-TB.

Male patients who were under active surveillance (i.e., with a prior positive biopsy) and those who underwent MRI prior to the release of the PI-RADS were excluded from the analysis. Those who underwent prostate procedures, including surgery (e.g., transurethral resection of the prostate) or radiation therapy, were also excluded. Furthermore, male patients with a region of interest of less than PI-RADS score of 3 who were administered oral $5 \alpha$-reductase inhibitors were excluded, as well as those who were deemed unsuitable by the research manager. Finally, a cohort of 104 men was obtained.

For each patient, we recorded the PSA level at biopsy, digital rectal examination findings, prostatic volume, overall PI-RADS and lesion scores, total number of biopsy cores obtained, presence of PCa, Gleason score (GS), and tumor infiltration. In this study, a GS $\geq$ 7 and/or a maximum cancer core length of $\geq 5 \mathrm{~mm}$ was considered as csPCa.

\section{Magnetic resonance imaging}

All male patients underwent a 1.5- or 3-Tesla prostate MRI. MRI imaging conditions were in accordance with the PI-RADS. Prior to biopsy, all suspicious lesions found on prostate MRI were scored by a single boardcertified radiologist with expertise in prostate imaging. If mpMRI was initially conducted and read by a thirdparty radiologist, a second reading was performed at our institution, and scoring was based on the PI-RADS guideline recommendations. Additionally, during the imaging evaluation, the radiologist was informed about patients' PSA levels, age, and other clinical information.

\section{Prostate biopsy}

For all biopsy procedures, the TRINITY ${ }^{\mathrm{TM}}$ system (Koelis, La Tronche, France) was used under spinal epidural anesthesia with the patient in the lithotripsy position. First, we visualized three-dimensional (3D) volume data obtained from MRI and real-time TRUS images. Elastic image fusion was conducted by semiautomatically contouring the MRI image of the entire prostate and suspected lesions on 3D TRUS images. A two-core biopsy targeted to each suspicious lesion, which was identified on MRI, was followed by a 14core SB. If there were three or more MRI lesions, two MRF-TBs were performed. In this case, one MRF-TB was conducted on the index lesion and another on the next suspected lesion. All biopsy cores were obtained by a single urologist.

\section{Histological evaluation}

The prostate biopsy specimens from each patient were assigned to four pathologists with special training, who then evaluated their assigned specimens.

\section{Statistical analysis}

We traditionally performed a 14-core SB, in which a 6-core was performed at the basal level (outside, center, inside on both sides), a 6-core at the mid-level (outside, center, inside on both sides), and a 2-core at the apex level (center on both sides) (Fig. 1). In this study, we compared how CDRs changed when the number of SBs used with MRF-TB was reduced. The CDR in the 10core $\mathrm{SB}$, excluding the biopsy results from the center of the base and from the mid-level on both sides, was evaluated. We used the Mann-Whitney $U$ test for comparison between the two groups. Wilcoxon's signed rank test was used to compare the CDR between a 14-core SB and a 10-core SB between the two corresponding groups. For each test result, a corresponding two-sided $P$ value of $<0.05$ was considered statistically significant. All analyses were performed using the SPSS software 

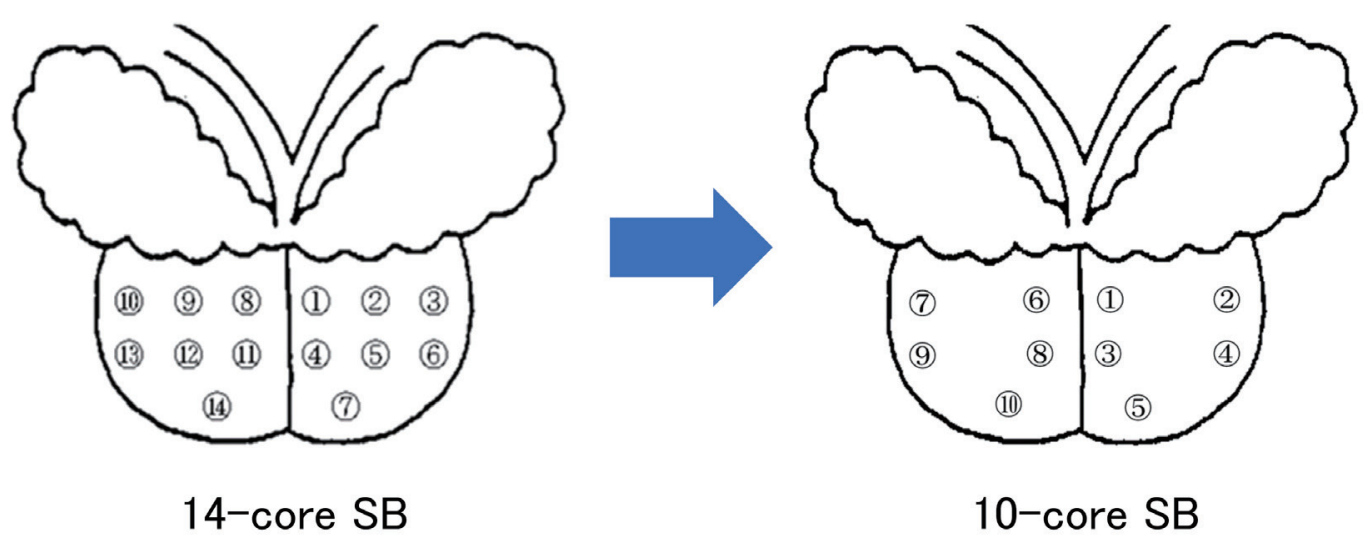

Fig. 1. Prostate biopsy site. 14-core SB: 6-core at base level (outside, center, inside on both sides), 6-core at mid-level (outside, center, inside on both sides), and 2-core at apex level (center on both sides). 10-core SB: 4-core at base level (outside, inside on both sides), 4-core at mid-level (outside, inside on both sides), and 2-core at apex level (center on both sides).

version 23.0 (IBM Corporation, Armonk, NY).

\section{RESULTS}

Table 1 presents the descriptive statistics of the study population. The study included 86 biopsy-naïve patients and 18 patients with a prior negative biopsy but with persistently high PSA levels. The median patient age was 70 years [interquartile range (IQR), 66-74 years]; the median PSA level was $8.62 \mathrm{ng} / \mathrm{mL}$ (IQR, 6.5-12.6 $\mathrm{ng} / \mathrm{mL}$ ); and the median prostate volume was $44 \mathrm{~mL}$ (IQR, 30.7-63.5 mL). All patients underwent simultaneous MRF-TB and a 14-core SB. Overall, 60 lesions were PI-RADS score of 3, 67 were PI-RADS score of 4, and 22 were PI-RADS score of 5. In total, 149 lesions were subjected to MRF-TB with 2-cores each. There were no significant prostate biopsy-related complications (Clavien-Dindo grade I) that required hospital admission in any of the patients.

\section{Patient level}

Figure 2 shows the overall CDR and csPCa CDR. The combination of $\mathrm{SB}$ and TB resulted in the highest CDR. Figure 3 shows the prostate CDR of the 14-core and 10core SB used in combination. The overall CDR was $55.8 \%$ for the former and $55.8 \%$ for the latter, thereby indicating that there was no significant difference $(P=$ $1.00)$ between the two. In addition, the CDR of csPCa was $51.9 \%$ for the former and $51.1 \%$ for the latter, which indicated that there was no significant difference $(P=$ 0.317 ) between the two.

\section{Lesion level}

In patients with a PI-RADS score of 3, the CDR was $20.0 \%$, the inflammation rate was $26.7 \%$, and the prostatic intraepithelial neoplasia (PIN) rate was $3.3 \%$. In those with a PI-RADS score of 4, the CDR was 56.7\%, the inflammation rate was $13.4 \%$, and the PIN rate was $4.5 \%$. In those with a PI-RADS score of 5 , the CDR was $77.3 \%$, the inflammation rate was $0.0 \%$, and the PIN rate was $0.0 \%$ (Table 2). The higher the PI-RADS score was, the higher the CDR. A PI-RADS score of $4 / 5$ resulted in a significant difference in CDR compared to a PI-RADS score of 3 (Fig. 4). Although patients with a PI-RADS score of 3 had a low CDR, this group also consisted of patients with a high GS (Fig. 5).

\section{DISCUSSION}

In this study, there was no significant difference in the CDR between the 14-core and 10-core SBs. Based on this result, our facility has reduced the number of biopsy cores in SB to 10. In addition, our facility has started conducting MRI-TRUS fusion biopsy. Furthermore, the combination of SB and MRF-TB resulted in the highest CDR in our study. These results were consistent with those of the other reports. Likewise, Calio et al. found that combining SB with MRF-TB resulted in a significantly reduced surgical GS upgrading compared with that of SB alone. ${ }^{6}$ Additionally, patients with a PI-RADS score of 3 had a low CDR. Nevertheless, there was a certain number of PCa cases with a high GS that necessitated proper precaution. Therefore, in patients with MRI lesions with a PI-RADS score of 3 or higher, we should consider performing MRF-TB.

In recent years, many studies have shown the effectiveness of MRF-TB. It was demonstrated that the csPCa detection rate of men undergoing MRF-TB was higher than that of men undergoing TRUS biopsy. ${ }^{7}$ Siddiqui et al. reported that in the prospective singlegroup cohort of 1,003 men, the number of high-risk cancers detected increased and the number of low-risk 
Table 1. Patients' characteristics

\begin{tabular}{lcc}
\hline No. patients & 104 & $(66-74)$ \\
Median age, years (IQR) & 70 & $(6.5-12.6)$ \\
Median ng/mL PSA (IQR) & 8.62 & $(30.7-63.5)$ \\
\hline Median ml prostate vol (IQR) & 44 & $(7-13)$ \\
\hline Median mm MRI index lesion diameter (IQR) & 10 & $(82.7)$ \\
\hline Prior systematic biopsy (\%): & 86 & $(14.4)$ \\
Naive & 15 & $(2.9)$ \\
\hline Prior negative & 3 & $(15.4)$ \\
\hline or Greater prior negative & 16 & $(40.3)$ \\
Abnormal digital rectal examination (\%) & & $(45.0)$ \\
\hline No. lesion PI-RADS (\%): & 60 & $(14.7)$ \\
\hline Score 3 & 67 & \\
\hline Score 4 & 22 & \\
\hline Score 5 & & \\
\hline No. biopsy core: & 1456 & \\
\hline SB & 298 & \\
\hline TB & & \\
\hline
\end{tabular}

Overall CDR

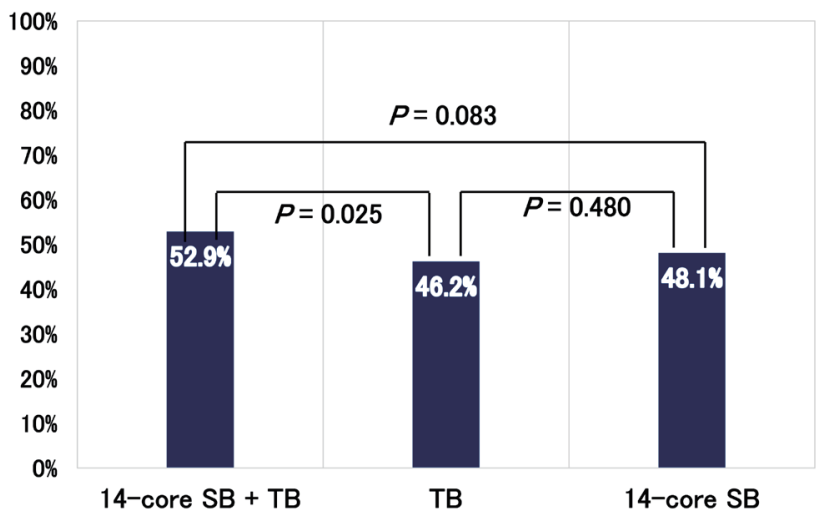

CDR of csPCa

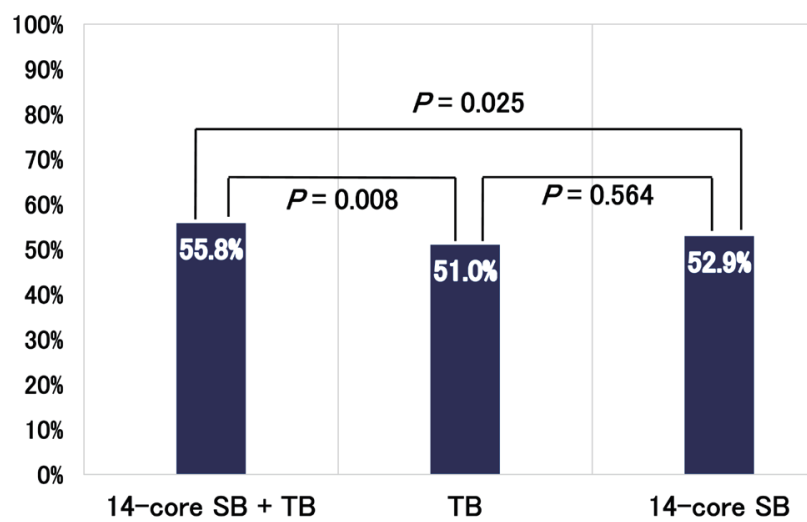

Fig. 2. The overall $\mathrm{CDR}$ and the $\mathrm{CDR}$ of csPCa. The CDR was compared for the combination of $\mathrm{SB}$ and $\mathrm{TB}$, and for TB alone and $\mathrm{SB}$ alone. We compared the overall CDR and the CDR of csPCa, respectively. The results showed that the combination of SB and TB had the highest CDR, but there was no significant difference in CDR between TB and the 14-core SB.

cancers detected decreased with the use of MRF-TB. ${ }^{8}$ Baco et al. reported comparable detection rates of csPCa between the 2-core MRF-TB and the 12-core SB. ${ }^{9}$ Ukimura et al. reported that the TRUS visibility of an MR-suspicious lesion facilitates image-guided biopsies, resulting in higher detection of $\mathrm{csPCa} .{ }^{10}$ Based on these recent studies, MRF-TB has the potential to be a gold standard diagnostic tool for men suspected of PCa. In addition, MRF-TB has made it possible to determine the GS and cancer localization of csPCa with high accuracy, making it easier to track the cancer progression of individual patients. The detailed information obtained by MRF-TB is expected to be applied to the accurate adaptation of active surveillance, surgical resection with improved curability, and nerve preservation, with further application in focal therapy. TRINITY ${ }^{\mathrm{TM}}$ records the 3D position information of the tissue collected by the biopsy and enables 3D display, making it easier to visualize where the cancer tissue is in the prostate. At our institution, in cases where robot-assisted laparoscopic 


\section{Overall CDR}

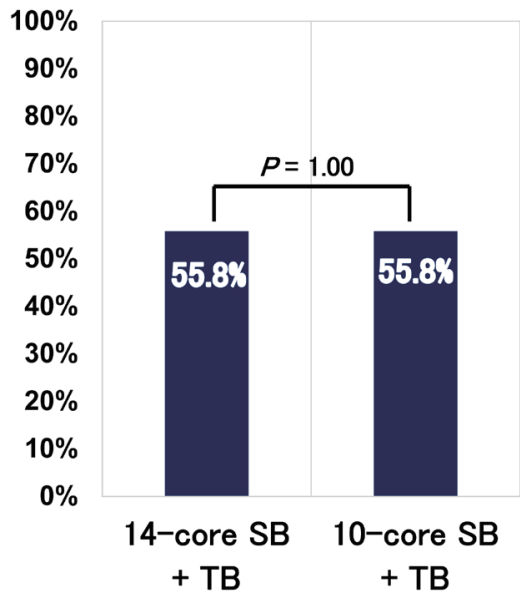

\section{CDR of csPCa}

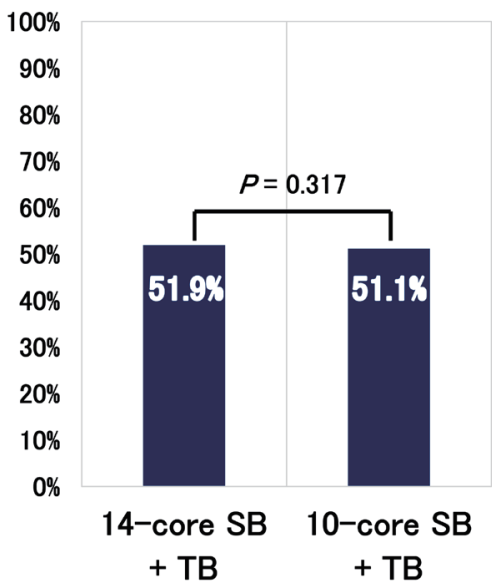

Fig. 3. The CDR by number of SB. A combination of 14-core SB and TB and 10-core SB and TB were compared with CDR. No significant differences were found in the overall CDR and CDR of csPCa.

Table 2. Histopathological results by PI-RADS score

\begin{tabular}{|c|c|c|c|c|c|c|}
\hline \multirow[b]{3}{*}{ No. MRI lesion } & \multicolumn{2}{|c|}{ PI-RADS } & \multicolumn{2}{|c|}{ PI-RADS } & \multicolumn{2}{|c|}{ PI-RADS } \\
\hline & \multicolumn{2}{|c|}{ score of 3} & \multicolumn{2}{|c|}{ score of 4} & \multicolumn{2}{|c|}{ score of 5} \\
\hline & 60 & $(20.0)$ & 67 & $(56.7)$ & 22 & (77.3) \\
\hline No. PCa detection (\%) & $12 / 60$ & & $38 / 67$ & & $17 / 22$ & \\
\hline $\mathrm{GS}=6$ & 4 & & 7 & & 1 & \\
\hline $\mathrm{GS}=7$ & 3 & & 23 & & 7 & \\
\hline $\mathrm{GS} \geq 8$ & 5 & & 8 & & 9 & \\
\hline No. inflammation (\%) & $16 / 60$ & $(26.7)$ & $9 / 67$ & (13.4) & $0 / 22$ & $(0.0)$ \\
\hline No. PIN (\%) & $2 / 60$ & (3.3) & $3 / 67$ & $(4.5)$ & $0 / 22$ & $(0.0)$ \\
\hline
\end{tabular}

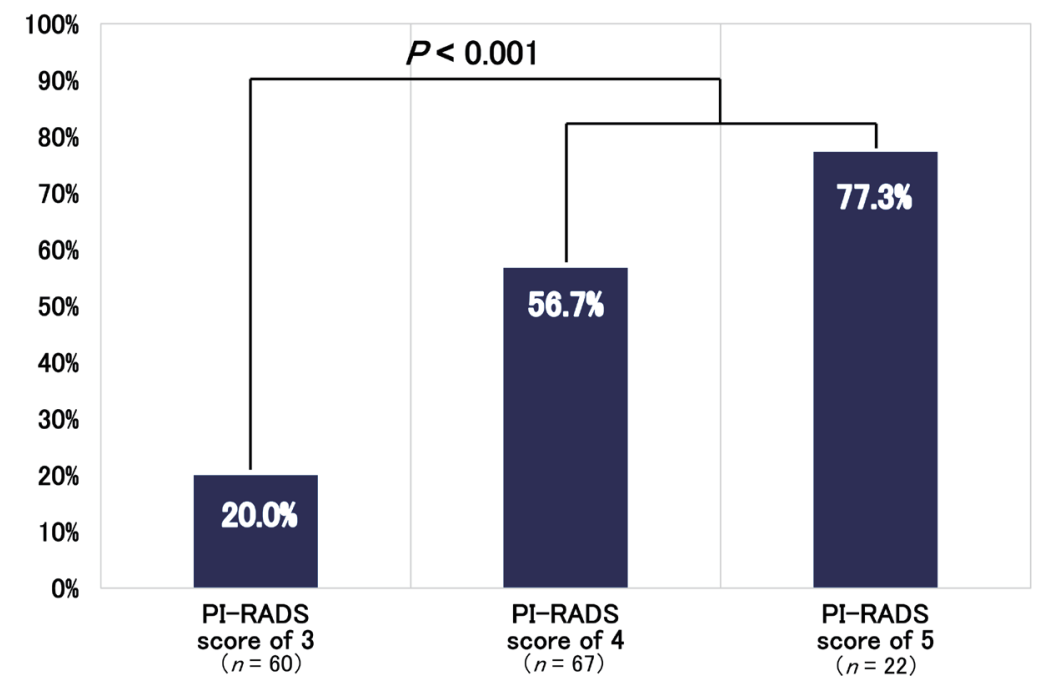

Fig. 4. The CDR by PI-RADS score. The CDR for PI-RADS score of 3 was $20.0 \%$, the CDR for score of 4 was $56.7 \%$, and the CDR for score of 5 was $77.3 \%$. Comparing score 3 and $4 / 5$, the CDR was significantly higher in the latter group $(P<0.001)$. 


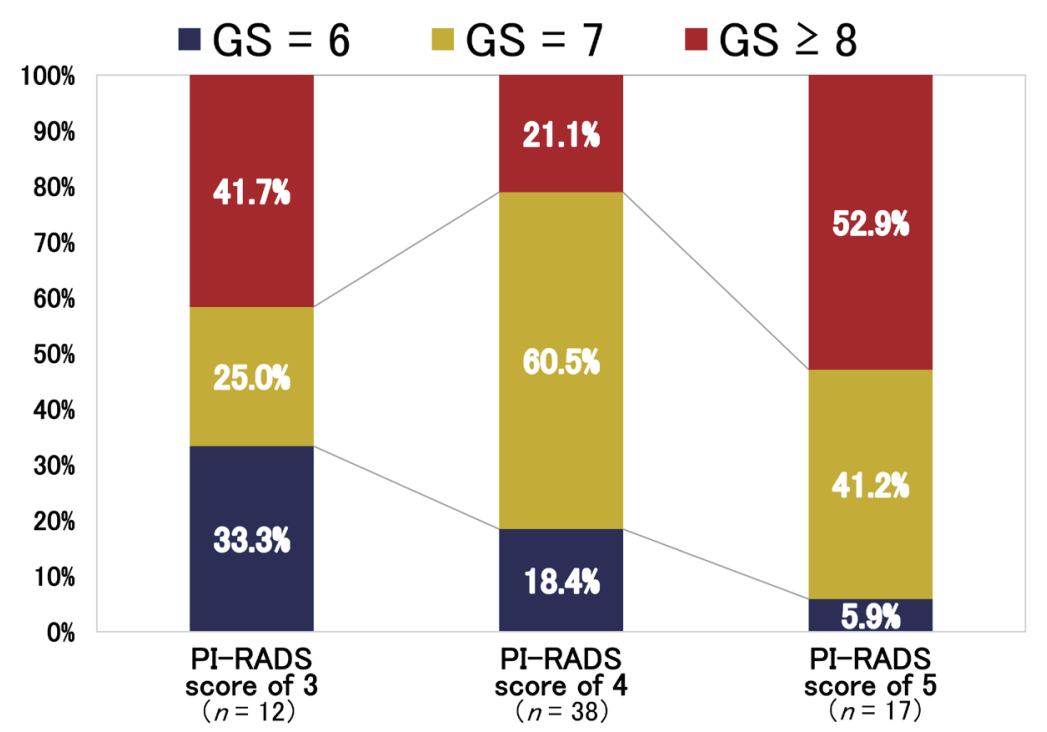

Fig. 5. GS assessment by PI-RADS score in prostate cancer-positive patients. PI-RADS score of 5 resulted in a high percentage of GS $\geq 8$. On the other hand, even with PI-RADS score of 3 , a certain percentage had GS $\geq 8$ findings.

radical prostatectomy is to be performed after MRF-TB, it is used to evaluate the localization of cancer in nervepreserving surgical selection. Moreover, we tried to visually improve the surgical precision by displaying a 3D model on the da Vinci Surgical System ${ }^{\mathrm{TM}}$.

This study compared the change in CDR, assuming that the number of SBs used in combination with MRF-TB was reduced from 14- to 10 cores in a retrospective study. Therefore, it was difficult to assess the substantial risks for complications and costs. In general, complications of prostate biopsy include hematuria, hematospermia, rectal bleeding, infection, pain, and urinary retention. Reducing the number of biopsy cores may lead to a reduction in these complications. However, the effect of the number of biopsy cores on hematuria was controversial. Ghani et al. reported that in a study involving 760 patients, there was no association between the incidence of hematuria and the number of biopsy cores ( $44 \%$ for 6 cores, $41 \%$ for 8 cores, and $39 \%$ for 12 cores). ${ }^{11}$ However, there was a study that found that an increase in the number of biopsy cores led to an increased incidence of hematuria. ${ }^{12}$ For rectal bleeding, McCormack et al. reported an association between the number of biopsy cores and anticoagulants. ${ }^{13}$ In addition, Ghani et al..$^{11}$ reported that the incidence of rectal bleeding was $17 \%$ in the 6-core, $26 \%$ in 8 -core, and $27 \%$ in 10 -core SB. These results suggested that the incidence of rectal bleeding was significantly increased as the number of biopsy cores increased. ${ }^{11}$ Regarding hematospermia, Berger et al. reported a significant association between the number of biopsy cores $(31.8 \%$ for 6 -cores, $37.4 \%$ for 10 -cores, and $38.4 \%$ for 15 -cores). ${ }^{14}$ In addition, some studies showed an association between the number of biopsy cores and the risk for pain during prostate biopsy. ${ }^{15-17}$ The risk for erectile dysfunction after prostate biopsy remains unclear. However, it might be related to a combination of psychological factors, such as the stress that a PCa diagnosis might confer. Nevertheless, repeated biopsies or extensive biopsies of the prostate might lead to erectile dysfunction. ${ }^{18}$ Further, there are no reports on the association between the number of biopsy cores and the risk for urinary retention. Additionally, a metaanalysis of infections did not show an association with the number of biopsy cores. ${ }^{15,19,20}$

There were a few reports on the cost efficiency of fusion biopsy in $\mathrm{PCa}$ care. Venderink et al. reported that fusion biopsy was superior to SB. ${ }^{21}$ In the clinical setting, it was important to distinguish csPCa from cisPCa. Onik et al. reported that the localization of csPCa can be determined via MRI with a $3 \mathrm{~mm}$ slice thickness; hence, mpMRI is efficient. ${ }^{22}$ If there is no obvious lesions noted on mpMRI, clinical follow-up without prostate biopsy can reduce the problem of overdiagnosis and overtreatment. ${ }^{23}$ mpMRI improves the cost-effectiveness of prostate biopsy; however, it should be noted that false negatives can occur in approximately $20 \%$ of cases. ${ }^{24,25}$ For the above reasons, MRF-TB can efficiently detect csPCa and may be cost-effective. However, studies on cost-effectiveness, which encompass the cost of diagnosis, reduction of overtreatment, and reduction in the number of biopsy cores, are limited. These findings 
require further validation. Furthermore, reliably detecting csPCa using MRF-TB only could reduce complications; however, this is not currently feasible because it has been reported that only $17.4 \%$ of cribriform tumors in pure form were visible on MRI. ${ }^{26}$ Therefore, with the current imaging diagnostic technology, performing MRF-TB only on lesions that are detected on MRI might result in the underdiagnosis of csPCa. Thus, the combination of SB and MRF-TB is considered essential. In fact, it has been reported that the false-negative rates of csPCa in targeted fusion prostate biopsy were $16.2 \%$ and $39.7 \%$ in patients with a PI-RADS score of 3 or greater and those with a PI-RADS score of 4 or greater, respectively. ${ }^{27}$ Although the effectiveness of MRF-TB has been reported previously, a study reported that there was a learning curve in establishing the MRF-TB procedure. Meng et al. reported that the csPCa detection rate increased by $26 \%(50 \%$ to $76 \%, P=0.025)$ with time in men with a PI-RADS score of 4 or 5 lesion. ${ }^{28}$ It was necessary to perform a certain number of cases to master the procedure impeccably. Furthermore, there is no clear standard for the number of MRF-TB cores to be collected for each MRI lesion. The number of TB cores is determined based on the judgment of the examiner, whose decision is based on the size and location of the lesion noted on MRI. According to Porpiglia et al., taking two cores at the center of the index lesion regardless of the diameter may provide a more accurate $\mathrm{CDR}$ and could optimize the chances of finding the highest Gleason pattern. ${ }^{29}$ On the other hand, there were skeptical opinions about the use of a 2-core TB. In fact, Dimitroulis et al. reported that the diagnostic utility did not change there were one or two cores used for the target lesion. ${ }^{2,30}$

Fusion biopsy results based on pre-biopsy MRI findings were effective in many cases. However, the significance of SB remains unclear, despite its standard utilization since PSA became popular. However, fusion biopsy is expensive and time-consuming compared with other conventional biopsy techniques. Currently, cognitive biopsy, which is less expensive and less timeconsuming, is often the diagnostic tool of choice in general clinical practice, except in large hospitals such as university hospitals. In the current Japanese medical system, fusion biopsy is billed at the same level as that of a general cognitive biopsy. This may explain why the fusion biopsy is not currently preferred. Nevertheless, the future trend may change.

This study confirmed the effectiveness of MRF-TB; however, there are some limitations. First, not all patients undergoing MRF-TB were diagnosed with PCa in this study. Furthermore, since not all patients diagnosed with PCa selected to undergo curative prostatectomy, comparison between the biopsy specimen and the whole prostate specimen was incomplete. For these reasons, it was not possible to reliably measure the standard parameters such as actual sensitivity, specificity, diagnostic accuracy, etc. This aspect is considered a major limitation of studies focusing on MRF-TB. Second, improvements in the PI-RADS and the collaboration among radiologists, pathologists, and urologists are important factors that have been previously shown to contribute to the enhancement of cancer detection over time. However, it is difficult to quantify these factors. ${ }^{31,32}$ Third, in this study, the comparison is based on the assumption that the number of biopsy cores has been reduced. Therefore, we did not compare actual biopsy results. Forth, when an examiner is performing $\mathrm{SB}$, he/she already has prior knowledge of suspicious lesions based on either US images or fusion MR images. Therefore, there was a possibility that bias occurred during random sampling.

Despite the limitations, our study has several strengths. First, at our facility, for all male patients who presented with a high PSA value, the possibility of selective bias can be reduced to a certain extent because we performed MRI prior to biopsy when medically possible. Furthermore, since the prostate biopsy was performed by a single examiner, we believe that stability of the procedure can be achieved.

In conclusion, the 2-core MRF-TB had the same $\mathrm{CDR}$ as that of the 14-core SB. The combination of MRF-TB and SB resulted in the highest CDR. However, there was no significant difference in the CDR when the number of SB cores to be used in combination was 14 and 10. Furthermore, it is still a matter of discussion as to whether these $10 \mathrm{SB}$ cores can be considered as optimal biopsy cores. We aim to conduct further investigation in the future with a larger the number of cases.

The authors declare no conflict of interest.

\section{REFERENCES}

1 Mouraviev V, Villers A, Bostwick DG, Wheeler TM, Montironi R, Polascik TJ. Understanding the pathological features of focality, grade and tumour volume of early-stage prostate cancer as a foundation for parenchyma-sparing prostate cancer therapies: active surveillance and focal targeted therapy. BJU Int. 2011;108:1074-85. DOI: 10.1111/j.1464410X.2010.10039.x, PMID: 21489116

2 Kasivisvanathan V, Rannikko AS, Borghi M, Panebianco V, Mynderse LA, Vaarala MH, et al.; PRECISION Study Group Collaborators. MRI-Targeted or Standard Biopsy for ProstateCancer Diagnosis. N Engl J Med. 2018;378:1767-77. DOI: 10.1056/NEJMoa1801993, PMID: 29552975 
3 Valerio M, Donaldson I, Emberton M, Ehdaie B, Hadaschik BA, Marks LS, et al. Detection of Clinically Significant Prostate Cancer Using Magnetic Resonance Imaging-Ultrasound Fusion Targeted Biopsy: A Systematic Review. Eur Urol. 2015;68:8-19. DOI: 10.1016/j.eururo.2014.10.026, PMID: 25454618

4 Arsov C, Rabenalt R, Blondin D, Quentin M, Hiester A, Godehardt E, et al. Prospective randomized trial comparing magnetic resonance imaging (MRI)-guided in-bore biopsy to MRI-ultrasound fusion and transrectal ultrasound-guided prostate biopsy in patients with prior negative biopsies. Eur Urol. 2015;68:713-20. DOI: 10.1016/j.eururo.2015.06.008, PMID: 26116294

5 Quentin M, Blondin D, Arsov C, Schimmöller L, Hiester A, Godehardt E, et al. Prospective evaluation of magnetic resonance imaging guided in-bore prostate biopsy versus systematic transrectal ultrasound guided prostate biopsy in biopsy naïve men with elevated prostate specific antigen. J Urol. 2014;192:1374-9. DOI: 10.1016/j.juro.2014.05.090, PMID: 24866597

6 Arsov C, Rabenalt R, Quentin M, Hiester A, Blondin D, Albers $\mathrm{P}$, et al. Comparison of patient comfort between MRguided in-bore and MRI/ultrasound fusion-guided prostate biopsies within a prospective randomized trial. World J Urol. 2016;34:215-20. DOI: 10.1007/s00345-015-1612-6, PMID: 26055645

7 Panebianco V, Barchetti G, Simone G, Del Monte M, Ciardi A, Grompone MD, et al. Negative Multiparametric Magnetic Resonance Imaging for Prostate Cancer: what's Next? Eur Urol. 2018;74:48-54. DOI: 10.1016/j.eururo.2018.03.007, PMID: 29566957

8 Siddiqui MM, Rais-Bahrami S, Turkbey B, George AK, Rothwax J, Shakir N, et al. Comparison of MR/ultrasound fusion-guided biopsy with ultrasound-guided biopsy for the diagnosis of prostate cancer. JAMA. 2015;313:390-7. DOI: 10.1001/jama.2014.17942, PMID: 25626035

9 Baco E, Rud E, Eri LM, Moen G, Vlatkovic L, Svindland A, et al. A Randomized Controlled Trial To Assess and Compare the Outcomes of Two-core Prostate Biopsy Guided by Fused Magnetic Resonance and Transrectal Ultrasound Images and Traditional 12-core Systematic Biopsy. Eur Urol. 2016;69:14956. DOI: 10.1016/j.eururo.2015.03.041, PMID: 25862143

10 Ukimura O, Marien A, Palmer S, Villers A, Aron M, de Castro Abreu AL, et al. Trans-rectal ultrasound visibility of prostate lesions identified by magnetic resonance imaging increases accuracy of image-fusion targeted biopsies. World J Urol. 2015;33:1669-76. DOI: 10.1007/s00345-015-1501-z, PMID: 25656687

11 Ghani KR, Dundas D, Patel U. Bleeding after transrectal ultrasonography-guided prostate biopsy: a study of 7-day morbidity after a six-, eight- and 12-core biopsy protocol. BJU Int. 2004;94:1014-20. DOI: 10.1111/j.1464-410X.2004.05096.x, PMID: 15541119

12 Chowdhury R, Abbas A, Idriz S, Hoy A, Rutherford EE, Smart JM. Should warfarin or aspirin be stopped prior to prostate biopsy? An analysis of bleeding complications related to increasing sample number regimes. Clin Radiol. 2012;67:e64-70. DOI: 10.1016/j.crad.2012.08.005, PMID: 22959852
13 McCormack M, Duclos A, Latour M, McCormack MH, Liberman D, Djahangirian O, et al. Effect of needle size on cancer detection, pain, bleeding and infection in TRUSguided prostate biopsies: a prospective trial. Can Urol Assoc J. 2012;6:97-101. DOI: 10.5489/cuaj.11169, PMID: 22511415

14 Berger AP, Gozzi C, Steiner H, Frauscher F, Varkarakis J, Rogatsch H, et al. Complication rate of transrectal ultrasound guided prostate biopsy: a comparison among 3 protocols with 6, 10 and 15 cores. J Urol. 2004;171:1478-81. DOI: 10.1097/01. ju.0000116449.01186.f7, PMID: 15017202

15 Saraçoğlu T, Unsal A, Taşkın F, Sevinçok L, Karaman CZ. The impact of pre-procedural waiting period and anxiety level on pain perception in patients undergoing transrectal ultrasound-guided prostate biopsy. Diagn Interv Radiol. 2012;18:195-9. PMID: 22042731

16 Alvarez-Múgica M, González Alvarez RC, Jalón Monzón A, Fernández Gómez JM, Rodríguez Faba O, Rodríguez Robles L, et al. [Tolerability and complications of ultrasound guided prostate biopsies with intrarectal lidocaine gel]. Arch Esp Urol. 2007;60:237-44. PMID: 17601298

17 Dell'atti L, Borea PA, Russo GR. Age: "a natural anesthetic" in pain perception during the transrectal ultrasound-guided prostate biopsy procedure. Urol J. 2011;78:257-61. DOI: 10.5301/RU.2011.8850, PMID: 22139799

18 Helfand BT, Glaser AP, Rimar K, Zargaroff S, Hedges J, McGuire BB, et al. Prostate cancer diagnosis is associated with an increased risk of erectile dysfunction after prostate biopsy. BJU Int. 2013;111:38-43. DOI: 10.1111/j.1464410X.2012.11268.x, PMID: 22639942

19 Paul R, Schöler S, van Randenborgh H, Kübler H, Alschibaja M, Busch R, et al. Morbidity of prostatic biopsy for different biopsy strategies: is there a relation to core number and sampling region? Eur Urol. 2004;45:450-6. DOI: 10.1016/ j.eururo.2003.12.007, PMID: 15041108

20 Irani J, Blanchet P, Salomon L, Coloby P, Hubert J, Malavaud $\mathrm{B}$, et al. Is an extended 20-core prostate biopsy protocol more efficient than the standard 12-core? A randomized multicenter trial. J Urol. 2013;190:77-83. DOI: 10.1016/j.juro.2012.12.109, PMID: 23313205

21 Venderink W, Govers TM, de Rooij M, Fütterer JJ, Sedelaar JPM. Cost-Effectiveness Comparison of Imaging-Guided Prostate Biopsy Techniques: Systematic Transrectal Ultrasound, Direct In-Bore MRI, and Image Fusion. AJR Am J Roentgenol. 2017;208:1058-63. DOI: 10.2214/AJR.16.17322, PMID: 28225639

22 Onik G, Miessau M, Bostwick DG. Three-dimensional prostate mapping biopsy has a potentially significant impact on prostate cancer management. J Clin Oncol. 2009;27:43216. DOI: $10.1200 / J C O .2008 .20 .3497$, PMID: 19652073

23 Washino S, Kobayashi S, Okochi T, Kameda T, Konoshi T, Miyagawa T, et al. Cancer detection rate of prebiopsy MRI with subsequent systematic and targeted biopsy are superior to non-targeting systematic biopsy without MRI in biopsy naïve patients: a retrospective cohort study. BMC Urol. 2018;18:51. DOI: 10.1186/s12894-018-0361-4, PMID: 29843694

24 Filson CP, Natarajan S, Margolis DJA, Huang J, Lieu P, Dorey FJ, et al. Prostate cancer detection with magnetic resonanceultrasound fusion biopsy: the role of systematic and targeted biopsies. Cancer. 2016;122:884-92. DOI: 10.1002/cncr.29874, PMID: 26749141 
25 Mortezavi A, Märzendorfer O, Donati OF, Rizzi G, Rupp NJ, Wettstein MS, et al. Diagnostic Accuracy of Multiparametric Magnetic Resonance Imaging and Fusion Guided Targeted Biopsy Evaluated by Transperineal Template Saturation Prostate Biopsy for the Detection and Characterization of Prostate Cancer. J Urol. 2018;200:309-18. DOI: 10.1016/ j.juro.2018.02.067, PMID: 29474846

26 Truong M, Feng C, Hollenberg G, Weinberg E, Messing EM, Miyamoto H, et al. A Comprehensive Analysis of Cribriform Morphology on Magnetic Resonance Imaging/Ultrasound Fusion Biopsy Correlated with Radical Prostatectomy Specimens. J Urol. 2018;199:106-13. DOI: 10.1016/j.juro.2017.07.037, PMID: 28728994

27 Pepe P, Garufi A, Priolo GD, Galia A, Fraggetta F, Pennisi M. Is it Time to Perform Only Magnetic Resonance Imaging Targeted Cores? Our Experience with 1,032 Men Who Underwent Prostate Biopsy. J Urol. 2018;200:774-8. DOI: 10.1016/j.juro.2018.04.061, PMID: 29679618

28 Meng X, Rosenkrantz AB, Huang R, Deng FM, Wysock JS, Bjurlin MA, et al. The Institutional Learning Curve of Magnetic Resonance Imaging-Ultrasound Fusion Targeted Prostate Biopsy: Temporal Improvements in Cancer Detection in 4 Years. J Urol. 2018;200:1022-9. DOI: 10.1016/ j.juro.2018.06.012, PMID: 29886090
29 Porpiglia F, De Luca S, Passera R, De Pascale A, Amparore D, Cattaneo G, et al. Multiparametric Magnetic Resonance/ Ultrasound Fusion Prostate Biopsy: Number and Spatial Distribution of Cores for Better Index Tumor Detection and Characterization. J Urol. 2017;198:58-64. DOI: 10.1016/ j.juro.2017.01.036, PMID: 28093292

30 Dimitroulis P, Rabenalt R, Nini A, Hiester A, Esposito I, Schimmöller L, et al. Multiparametric Magnetic Resonance Imaging/Ultrasound Fusion Prostate Biopsy-Are 2 Biopsy Cores per Magnetic Resonance Imaging Lesion Required? J Urol. 2018;200:1030-4. DOI: 10.1016/j.juro.2018.05.002, PMID: 29733837

31 Rosenkrantz AB, Ayoola A, Hoffman D, Khasgiwala A, Prabhu V, Smereka P, et al. The Learning Curve in Prostate MRI Interpretation: Self-Directed Learning Versus Continual Reader Feedback. AJR Am J Roentgenol. 2017;208:W92-100. DOI: 10.2214/AJR.16.16876, PMID: 28026201

32 Rosenkrantz AB, Lim RP, Haghighi M, Somberg MB, Babb JS, Taneja SS. Comparison of interreader reproducibility of the prostate imaging reporting and data system and likert scales for evaluation of multiparametric prostate MRI. AJR Am J Roentgenol. 2013;201:W612-8. DOI: 10.2214/ AJR.12.10173, PMID: 24059400 